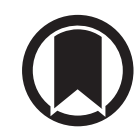

CrossMark

\title{
Joint effect of airflow limitation and emphysema on postoperative outcomes in early-stage nonsmall cell lung cancer
}

\author{
Sumin Shin ${ }^{1}$, Hye Yun Park ${ }^{2,7}$, Hyunkyung Kim³ ${ }^{3}$ Hong Kwan Kim', \\ Yong Soo Choi ${ }^{1}$, Jhingook Kim¹, Sang-Won Um², Myung Jin Chung ${ }^{4}$, \\ Hojoong Kim², 0 Jung Kwon², Jae Ill Zo ${ }^{1}$, Eliseo Guallar ${ }^{3,5}$, Juhee Cho ${ }^{3,5,6}$ and \\ Young Mog Shim ${ }^{1,7}$
}

Affiliations: ${ }^{1}$ Dept of Thoracic and Cardiovascular Surgery, Samsung Medical Center, Sungkyunkwan University School of Medicine, Seoul, South Korea. ${ }^{2}$ Division of Pulmonary and Critical Care Medicine, Dept of Medicine, Samsung Medical Center, Sungkyunkwan University School of Medicine, Seoul, South Korea. ${ }^{3}$ Center for Clinical Epidemiology, Samsung Medical Center, Sungkyunkwan University School of Medicine, Seoul, South Korea. ${ }^{4}$ Dept of Radiology, Samsung Medical Center, Sungkyunkwan University School of Medicine, Seoul, South Korea. ${ }^{5}$ Depts of Epidemiology and Medicine, and Welch Center for Prevention, Epidemiology, and Clinical Research, Johns Hopkins Bloomberg School of Public Health, Baltimore, MD, USA. ${ }^{6}$ Dept of Clinical Research Design and Evaluation, SAIHST, Sungkyunkwan University, Seoul, South Korea. ${ }^{7}$ Both authors contributed equally.

Correspondence: Hye Yun Park, Division of Pulmonary and Critical Care Medicine, Dept of Medicine, Samsung Medical Center, Sungkyunkwan University School of Medicine, 81 Irwon-ro, Gangnam-gu, Seoul, 06351, Korea. E-mail: hyeyunparkaskku.edu

ABSTRACT This study aims to evaluate the joint effect of severity of airflow limitation and emphysema on postoperative pulmonary complications (PPCs) and overall survival after complete resection in patients with early-stage nonsmall cell lung cancer (NSCLC).

We retrospectively studied 413 male patients with pathologic stage I or II NSCLC between 2007 and 2009. Severity of airflow limitation was defined based on forced expiratory volume in $1 \mathrm{~s}$. Emphysema was defined by $\geqslant 5 \%$ low attenuation area at $-950 \mathrm{HU}$.

In multivariable-adjusted analyses, the adjusted odds ratio (aOR) for any PPC, comparing patients with moderate-to-severe airflow limitation to those without airflow limitation, was 2.23 , and the aOR comparing patients with emphysema to those without emphysema was 1.77 . However, the joint effect of airflow limitation and emphysema was much higher than expected from the independent effects of both factors (aOR 8.90). Moreover, patients with coexisting moderate-to-severe airflow limitation and emphysema had significantly poorer overall survival than any other group.

Patients with moderate-to-severe airflow limitation and emphysema had almost nine times the risk of PPCs and poorer survival than patients with neither of these conditions. Integrated assessment of airflow limitation severity and emphysema is necessary for the optimal selection of candidates for lung resection surgery of early-stage NSCLC.

@ERSpublications

Postoperative outcomes after complete resection in early-stage NSCLC depends on both airflow limitation and emphysema http://ow.ly/fAjl3049YKn

This article has supplementary material available from erj.ersjournals.com

Received: June 102016 | Accepted after revision: Sept 032016 | First published online: Nov 032016

Support statement: This study was supported by Samsung Medical Center Foundation for Medical Research (SMO1140211). Funding information for this article has been deposited with the Open Funder Registry.

Conflict of interest: None declared.

Copyright @ERS 2016 


\section{Introduction}

Chronic obstructive pulmonary disease (COPD) is characterised by persistent irreversible airflow obstruction with enhanced inflammation in the airways and lung parenchyma [1]. In addition, COPD is associated with systemic inflammation, with numerous extrapulmonary manifestations and comorbidities, including the development of primary lung cancer [2,3]. The risk of lung cancer in COPD patients is up to five-fold higher than that of smokers without COPD, independent of age, sex and smoking history [3-6]. COPD patients have an increased risk of pulmonary complications and poorer survival outcomes after lung cancer surgery compared to those without COPD [7-9]. Thus, limited resections or nonsurgical therapeutic options are more often considered for COPD patients.

However, COPD is a heterogeneous disease with different degrees of airflow limitation and emphysema. The presence and extent of emphysema varies greatly, even in COPD patients with a similar degree of airflow limitation [10]. Moreover, treatment response to bronchodilators varies depending on the combination of severity of airflow limitation and emphysema [11]. So far, several studies have evaluated the impact of severity of airflow limitation or the presence of emphysema on surgical outcomes [12-15]. The presence of emphysema upon computed tomography (CT) adversely affected both overall and disease-specific survival after early-stage lung cancer surgery [12]. A recent study in stage I nonsmall cell lung cancer (NSCLC) patients found that severity of airflow limitation was negatively associated with long-term survival after lobectomy [15]. However, these studies evaluted the effects of airflow limitation and emphysema independent from one another and did not consider their joint effect on postoperative outcomes after lung cancer surgery. Thus, this study aims to evaluate the joint effect of airflow limitation severity and emphysema on postoperative pulmonary complications and overall survival after complete resection in early-stage NSCLC patients.

\section{Patients and methods}

\section{Study population}

We conducted a retrospective cohort study of male patients who underwent complete resection for stage I and II NSCLC and spirometry and CT scanning prior to surgery at a university-based tertiary hospital in Seoul, Korea from January 2007 to December 2009 ( $n=430)$. We excluded patients with multiple primary lung cancers $(n=2)$, previous pulmonary resection $(n=2)$, concomitant idiopathic pulmonary fibrosis or fibrotic nonspecific interstitial pneumonia $(n=7)$ and uncontrolled other malignancies $(n=6)$. The final sample included 413 patients. This study was approved by the institutional review board of Samsung Medical Center, which exempted the requirement for informed consent, as we only used deidentified data collected as part of routine clinic visits.

\section{Measurements}

Preoperative staging workup

Clinical characteristics including age at diagnosis, smoking status, body mass index (BMI), comorbidity, tumour histology, pathologic stage and type of surgery were obtained from electronic medical records. The routine preoperative workup included spirometry, CT scans of the chest and upper abdomen, positron emission tomography (PET)/CT scans, flexible bronchoscopy and magnetic resonance imaging of the brain. Spirometry was performed using Vmax 22 spirometers (SensorMedics, Yorba Linda, CA, USA) according to the American Thoracic Society/European Respiratory Society criteria [16]. Airflow limitation was based on a ratio of forced expiratory volume in $1 \mathrm{~s}(\mathrm{FEV} 1)$ to forced vital capacity (FVC) of $<0.70$. Severity of airflow limitation was classified as follows: mild airflow limitation $\mathrm{FEV}_{1} \geqslant 80 \%$ predicted; moderate-to-severe airflow limitation $\mathrm{FEV}_{1}<80 \%$ pred. Because only two patients had severe airflow limitation, we included these patients in the moderate-to-severe airflow limitation group.

Emphysema severity was quantified using CT using available software (Extended Brilliance Workspace v3.0; Philips Medical Systems, Cleveland, OH, USA). The presence of emphysema was defined as low attenuation area at $-950 \mathrm{HU} \geqslant 5 \%[12,17,18]$. We generated six airflow limitation emphysema groups based on the combination of airflow limitation severity and the presence of emphysema as follows: 1) no airflow limitation and no emphysema; 2) no airflow limitation and emphysema; 3 ) mild airflow limitation and no emphysema; 4) mild airflow limitation and emphysema; 5) moderate-to-severe airflow limitation and no emphysema; and 6) moderate-to-severe airflow limitation and emphysema.

For patients with preoperatively proven NSCLC, cervical mediastinoscopy or endobronchial ultrasoundguided transbronchial needle aspiration was performed to exclude N2 disease. Patients were staged according to the 7th edition of the TNM classification [19].

\section{Postoperative pulmonary complications}

We considered the following postoperative pulmonary complications (PPCs) as study outcomes: pneumonia, acute respiratory distress syndrome (ARDS), significant atelectasis requiring bronchoscopy or reintubation, 
empyema, bronchopleural fistula and prolonged air leakage during hospitalisation or readmission for 60 days postoperatively. Detailed criteria for each PPC are described in online supplementary table S1. In addition, we considered a combined outcome including all PPCs.

Postoperative treatment and follow-up

Adjuvant chemotherapy, radiotherapy or concurrent chemoradiotherapy were administered to patients with stage II disease if they were able to tolerate the additional treatments. Patients were regularly evaluated using chest CT scans every 3-4 months for the first 2 years after surgery and every 6 months thereafter, and by annual PET/CT scans for detection of recurrence. Overall survival was defined as the time from the date of surgery until the last date of follow-up for patients who remained alive or until death.

\section{Statistical analysis}

The independent and joint effects of airflow limitation severity and emphysema on PPCs were evaluated using multivariate logistic regression. Models were adjusted for age at diagnosis, smoking status, pathologic stage, histology, comorbidity and type of surgery. Comorbidities included diabetes, hypertension, cardiovascular disease (ischaemic heart disease, congestive heart failure and arrhythmia) or bronchiectasis. Survival analysis methods, including Kaplan-Meier methods and the log-rank tests were used to evaluate the joint effect of airflow limitation and emphysema on overall survival. All statistical analyses were two-sided with a significance level set at 0.05. Analyses were performed using STATA version 13 (StataCorp LP, College Station, TX, USA).

\section{Results}

The mean age of patients studied was 63.6 years and only $5.8 \%$ of the patients were never-smokers. 108 (35.4\%) patients developed at least one PPC (table 1).

Compared to patients without PPCs, those who developed PPCs were more likely to have squamous cell carcinoma and lower FEV1 and FVC. Pathologic stage was not different between the groups. Prolonged air leakage ( $n=54$, or $50.0 \%$ of patients with PPCs) and pneumonia $(n=37,34.3 \%)$ were the two most frequent PPCs, followed by significant atelectasis $(n=15,13.9 \%)$. Patients with no airflow limitation and no emphysema were the least likely to develop PPCs, while patients with moderate-to-severe airflow limitation and emphysema were the most likely to develop PPCs $(19.3 \%$ versus $66.8 \%, \mathrm{p}<0.001$; table 2$)$. Specifically, the rates of prolonged air leakage $(38.1 \%)$ and pneumonia/ARDS $(38.1 \%)$ were significantly higher in patients with moderate-to-severe airflow limitation and emphysema compared to any other groups.

In multivariable-adjusted analyses, the OR (95\% CI) for any PPC comparing patients with moderate-to-severe airflow limitation to those with no airflow limitation was 2.23 (1.11-4.46), while for those comparing patients with emphysema to those without emphysema it was 1.77 (1.05-2.96). However, the joint effect of airflow limitation and emphysema was much higher than expected from the independent effects of both factors $(8.90$ (3.02-26.19); table 3).

With respect to survival, two patients died within 30 days of surgery in the moderate-to-severe airflow limitation and emphysema group (9.5\%) and two patients died in all the other groups (2.7\%; $\mathrm{p}=0.02$; table 2$)$, although after 90 days the differences were no longer statistically significant. Kaplan-Meier curves showed that the severity of airflow limitation and the presence of emphysema were both associated with poor survival (online supplementary figures S1 and S2), but patients with both moderate-to-severe airflow limitation and emphysema had still significantly poorer survival than any other group (figure 1).

\section{Discussion}

In this longitudinal cohort study of early-stage NSCLC patients, we found that the risk of having PPCs increased up to 8.9-fold when patients had moderate-to-severe airflow limitation and emphysema concomitantly, compared to patients with neither airflow limitation nor emphysema. Patients with both moderate-to-severe airflow limitation and emphysema also had significantly poorer overall survival than patients without both conditions. To our knowledge, this is the first study to evaluate the joint effect of airflow limitation and emphysema on PPCs and overall survival after complete resection in early-stage NSCLC patients. Our findings support a substantially increased risk of PPCs and poor overall survival of NSCLC patients after complete resection when the patient has coexisting moderate-to-severe airflow limitation and emphysema.

The effect of airflow limitation on postoperative outcomes in NSCLC patients has been evaluated in prior studies. A study of 1461 patients who had undergone pulmonary resection for lung cancer from 1990 to 2005 found that those with higher airflow limitation grades had higher rates of PPCs and poorer long-term survival [13]. However, this study included patients with advanced pathologic stages, which is a well-known prognostic factor regardless of the presence of COPD. In addition, patients with advanced-stage disease received chemotherapy or radiotherapy, which might influence PPCs and overall survival. In our study, we restricted the study to early-stage NSCLC patients who underwent complete anatomic resection to allow 
TABLE 1 Characteristics of the study population

\begin{tabular}{|c|c|c|c|c|}
\hline & All & PPCs & Non-PPCs & p-value \\
\hline Subjects $\mathrm{n}$ & 413 & 108 & 305 & \\
\hline Age years & $63.6 \pm 9.3$ & $65.87 \pm 8.43$ & $62.86 \pm 9.50$ & $<0.001$ \\
\hline Body mass index $\mathrm{kg} \cdot \mathrm{m}^{-2}$ & $23.3 \pm 3.1$ & $21.93 \pm 3.50$ & $23.81 \pm 2.80$ & $<0.001$ \\
\hline Smoking status & & & & 0.01 \\
\hline Current or ex-smoker & 389 (94.2) & $107(99.1)$ & $282(92.5)$ & \\
\hline Never-smoker & $24(5.8)$ & $1(1.0)$ & $23(7.5)$ & \\
\hline \multicolumn{5}{|l|}{ Comorbidities } \\
\hline Hypertension" & $149(36.1)$ & $33(30.6)$ & $116(38.0)$ & 0.16 \\
\hline Diabetes & 64 (15.5) & $11(10.2)$ & $53(17.4)$ & 0.08 \\
\hline Cardiovascular disease $e^{\pi}$ & $31(7.5)$ & $3(2.8)$ & $28(9.2)$ & 0.03 \\
\hline Histology & & & & 0.03 \\
\hline Squamous cell carcinoma & $207(50.1)$ & $65(60.2)$ & $142(46.6)$ & \\
\hline Adenocarcinoma & $171(41.4)$ & $33(30.6)$ & $138(45.3)$ & \\
\hline Others & 35 (8.5) & $10(9.3)$ & $25(8.2)$ & \\
\hline Pathologic stage & & & & 0.07 \\
\hline IA & $106(25.7)$ & $18(16.7)$ & $88(28.6)$ & \\
\hline IB & 148 (35.8) & $40(37.0)$ & $108(35.4)$ & \\
\hline$\| A$ & $86(20.8)$ & $26(24.1)$ & $60(19.7)$ & \\
\hline IIB & $73(17.7)$ & $24(22.2)$ & $49(16.1)$ & \\
\hline Type of surgery & & & & $<0.001$ \\
\hline Lobectomy & 353 (85.5) & $87(80.6)$ & $266(87.2)$ & \\
\hline Bilobectomy & 33 (8.0) & $17(15.7)$ & $16(5.3)$ & \\
\hline Pneumonectomy & $27(6.5)$ & $4(3.7)$ & $23(7.5)$ & \\
\hline \multicolumn{5}{|l|}{ Preoperative PFT } \\
\hline FVC L & $3.8 \pm 0.7$ & $3.7 \pm 0.6$ & $3.9 \pm 0.7$ & $<0.001$ \\
\hline FVC $\%$ pred & $99 \pm 14$ & $98 \pm 13$ & $100 \pm 15$ & 0.17 \\
\hline FEV $1 \mathrm{~L}$ & $2.6 \pm 0.6$ & $2.4 \pm 0.5$ & $2.7 \pm 0.6$ & $<0.001$ \\
\hline FEV $1 \%$ pred & $96 \pm 17$ & $90.7 \pm 17.9$ & $97 \pm 16$ & $<0.001$ \\
\hline $\mathrm{FEV}_{1} / \mathrm{FVC} \%$ & $68 \pm 10$ & $65.1 \pm 11.6$ & $69 \pm 9$ & $<0.001$ \\
\hline Airflow limitation & & & & $<0.001$ \\
\hline None & $196(47.5)$ & 41 (38.0) & $155(50.8)$ & \\
\hline Mild & $161(39.0)$ & $43(39.8)$ & $118(38.7)$ & \\
\hline Moderate-to-severe ${ }^{+}$ & $56(13.6)$ & $24(22.2)$ & 32 (10.5) & \\
\hline Emphysema \% & $3.7 \pm 4.5$ & $4.7 \pm 4.9$ & $3.4 \pm 4.4$ & $<0.001$ \\
\hline Emphysema $\geqslant 5 \%$ & $103(24.9)$ & $38(35.2)$ & 65 (21.3) & $<0.001$ \\
\hline Bronchiectasis & $17(4.1)$ & $9(8.3)$ & $8(2.6)$ & 0.02 \\
\hline
\end{tabular}

Data are presented as $n$, mean \pm SD $n(\%)$, unless otherwise stated. PPCs: postoperative pulmonary complications; PFT: pulmonary function test; FVC: forced vital capacity; FEV1: forced expiratory volume in $1 \mathrm{s.}{ }^{\#}$ : arterial hypertension; " : includes ischaemic heart disease, congestive heart failure and arrhythmia; ${ }^{+}$: only two patients had severe airflow limitation.

assessment of the independent impact of airflow limitation severity on surgical outcomes. Patients with moderate-to-severe airflow limitation were more likely to have PPCs such as pneumonia/ARDS and prolonged air leakage after complete surgical resection of early-stage NSCLC. Severity of airflow limitation was also associated with poorer overall survival, supporting the findings of previous studies [14-15].

The presence of emphysema is associated with lung cancer diagnosis [20, 21]. Our study further demonstrates an independent association of emphysema with PPCs and overall survival. Emphysema is a risk factor for developing air leak in patients undergoing thoracic surgery, and prolonged air leak is a common complication after lung volume reduction surgery for emphysema treatment [22]. Consistent with these findings, patients with emphysema in the present study were more likely to have prolonged air leakage as well as pneumonia/ARDS after pulmonary resection for early-stage NSCLC, probably because the lung tissue surrounding emphysema is more easily damaged during surgery and could take longer to recover.

The joint effect of moderate-to-severe airflow limitation and emphysema after complete resection among early-stage NSCLC patients was higher than expected from multiplicative effects of moderate-to-severe airflow limitation and emphysema, as patients with moderate-to-severe airflow limitation and emphysema had approximately nine times higher risk of PPCs compared to patients with neither of these conditions. Consistently, the moderate-to-severe airflow limitation patients with emphysema had higher 30-day mortality rate than any other airflow limitation-emphysema groups. Therefore, prudent consideration of 
TABLE 2 Postoperative pulmonary complications (PPCs) and mortality according to airflow limitation and emphysema

\begin{tabular}{|c|c|c|c|c|c|c|c|c|}
\hline & Subjects $\mathrm{n}$ & Any PPC & Pneumonia/ARDS & Empyema/BPF & Prolonged air leakage & Significant atelectasis ${ }^{\#}$ & 30 days & 90 days \\
\hline Total & 413 & $108(26.2)$ & $37(34.3)^{\pi}$ & $6(5.6)^{\pi}$ & $54(50.0)^{\pi}$ & $15(13.9)^{\pi}$ & $4(1.0)$ & $10(2.4)$ \\
\hline \multicolumn{9}{|l|}{ AL-emphysema } \\
\hline No AL-no emphysema & 161 & $31(19.3)$ & $10(6.2)$ & $1(0.6)$ & $17(10.6)$ & $4(2.5)$ & $1(0.6)$ & $4(2.5)$ \\
\hline No AL-emphysema & 35 & $10(28.6)$ & $3(8.6)$ & $0(0)$ & $4(11.4)$ & $3(8.6)$ & 0 & $1(2.9)$ \\
\hline Mild AL-no emphysema & 114 & $29(25.4)$ & $10(8.8)$ & $3(2.6)$ & 12 (10.5) & 6 (5.3) & 0 & $2(1.8)$ \\
\hline Mild AL-emphysema & 47 & 14 (29.8) & $4(8.5)$ & $1(2.1)$ & $8(17.0)$ & $1(2.1)$ & $1(2.1)$ & $1(2.1)$ \\
\hline Moderate-to-severe $\mathrm{AL}^{+}$-no emphysema & 35 & $10(28.6)$ & $2(5.7)$ & $1(2.9)$ & $5(14.3)$ & $1(2.9)$ & 0 & 0 \\
\hline Moderate-to-severe $\mathrm{AL}^{+}$-emphysema & 21 & $14(66.8)$ & $8(38.1)$ & $0(0)$ & $8(38.1)$ & $0(0)$ & $2(9.5)$ & $2(9.5)$ \\
\hline p-value & & $<0.001$ & $<0.001$ & 0.53 & 0.04 & $<0.001$ & 0.02 & 0.35 \\
\hline
\end{tabular}

Data are presented as $\mathrm{n}(\%)$, unless otherwise stated. ARDS: acute respiratory distress syndrome; BPF: bronchopleural fistula; AL: airflow limitation. \#: cases requiring therapeutic bronchoscopy or reintubation to control the atelectasis; cases accompanying pneumonia or ARDS were not included; " : the denominator for percentages was the number of patients with any postoperative pulmonary complication $(n=108) ;{ }^{+}$: only two patients had severe AL. 
TABLE 3 Multivariate analysis for independent and joint effect of airflow limitation (AL) and emphysema

Adjusted OR $(95 \% \mathrm{Cl})$

p-value

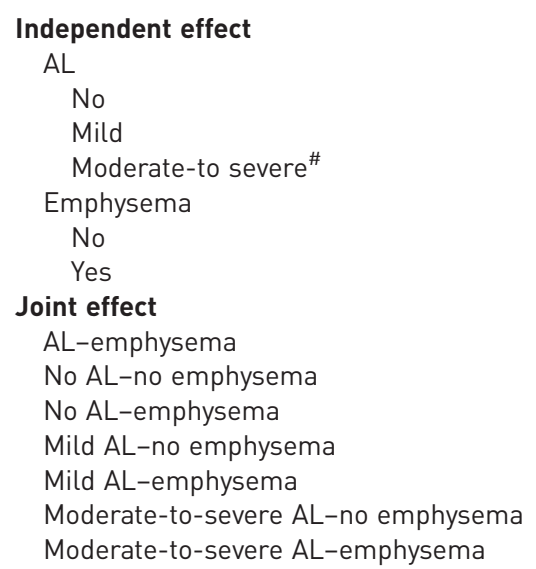

1 (ref.)

$\begin{array}{cc}0.93(0.53-1.61) & 0.79 \\ 2.23(1.11-4.46) & 0.02 \\ 1 \text { (ref.) } & \\ 1.77(1.05-2.96) & 0.03 \\ & \\ 1 \text { (ref.) } & \\ 1.76(0.73-4.23) & 0.21 \\ 1.10(0.58-2.06) & 0.77 \\ 1.12(0.50-2.51) & 0.78 \\ 1.30(0.53-3.21) & 0.57 \\ 8.90(3.02-26.19) & <0.001\end{array}$

Both independent and joint model were adjusted for age at diagnosis, smoking status, pathologic stage, histology, comorbidity and type of surgery. Comorbidities included diabetes, hypertension, cardiovascular disease or bronchiectasis. " : only two patients had severe airflow limitation.

both airflow limitation severity and emphysema is necessary for the selection of candidates for early-stage NSCLC surgery. Although several mechanisms may underlie the increased risk associated with the combined presence of airflow limitation and emphysema, decreased fat free mass (FFM) may play a role in increasing the risk of PPCs and mortality. A decreased FFM index was associated with worse outcomes of COPD [23, 24], which was connected with increased resting energy expenditure, deconditioning and elevated levels of systemic inflammation $[23,25]$. Indeed, in our study, patients with lower BMI were more likely to have PPCs than patients with higher BMI. Further studies with a larger sample size and with FFM measures are necessary to determine the mechanisms underlying the increased risk associated with the combined presence of airflow limitation and emphysema.

In our study, mild airflow limitation was not independently associated with an increased risk of PPCs or poor overall survival. This supports our current understanding of COPD, emphasising the impact of

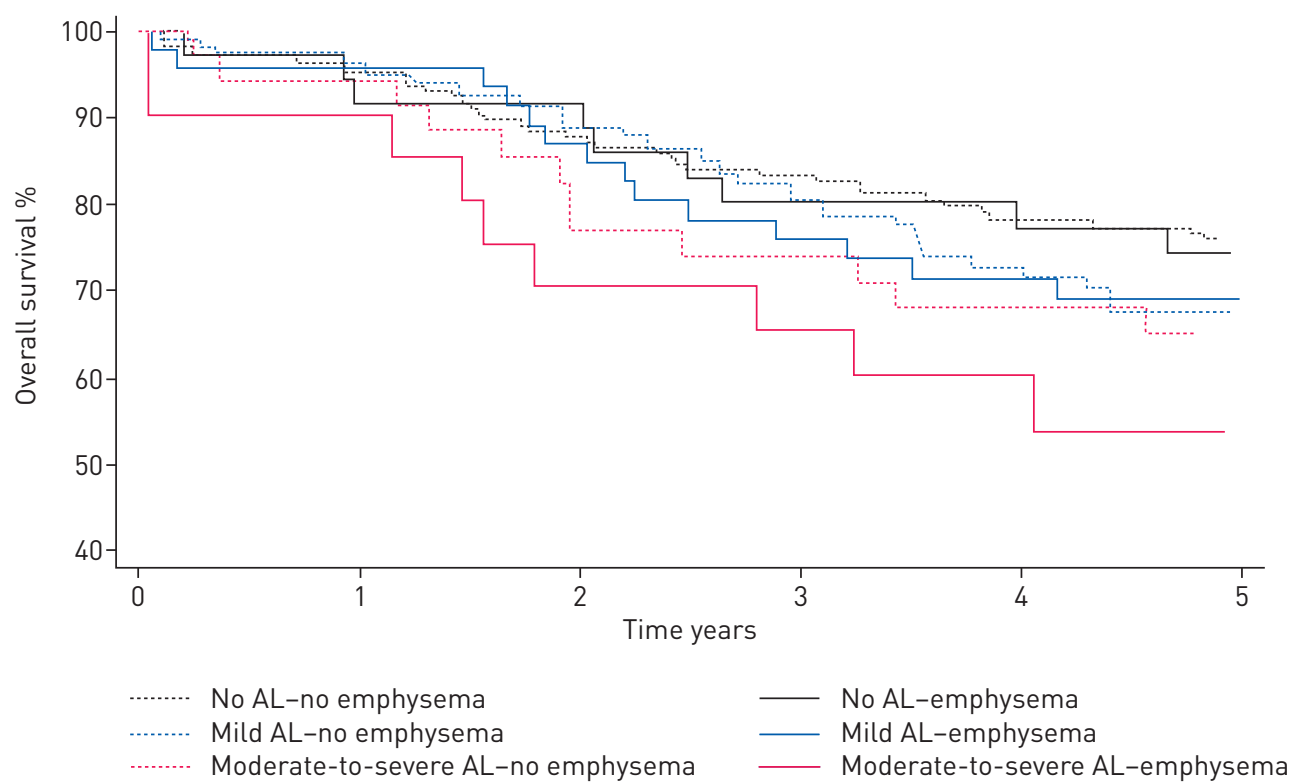

FIGURE 1 Overall survival according to chronic obstructive pulmonary disease (airflow limitation (AL))emphysema. 
moderate-to-severe grades of airflow limitation on treatment and prognosis [3]. Given the high incidence of lung cancer among patients with mild COPD [20], the results of our study provide important therapeutic reassurance about the safety of complete surgical resection, which may be the best treatment option for early-stage NSCLC patients with mild COPD.

There are several limitations to this study. First, since airflow limitation was based on prebronchodilator pulmonary function tests [26], some patients classed normal after post-bronchodilator pulmonary function tests could have been included in the mild airflow limitation group, and some mild airflow limitation patients could have been included in the moderate airflow limitation group. While this type of misclassification would tend to dilute the study findings, we were still able to observe a significant impact of the airflow limitation severity and emphysema. Also, this study was conducted only in male patients at a tertiary hospital and the results of the study might not be generalisable to female patients or to different settings. However, rates of female smoking are very low in Korea [27], and most female patients underwent surgical resection for pulmonary nodules with ground-glass opacity, which may influence lung cancer survival [28].

In conclusion, the risk of PPCs and overall survival after complete resection in early-stage NSCLC patients depended on both airflow limitation and the presence of emphysema, and the joint effect of moderate-to-severe airflow limitation and emphysema increased the risk of PPCs by almost nine-fold compared to patients with neither of the conditions. Further integrated assessment of airflow limitation severity and emphysema is necessary in order to achieve optimal surgical outcomes after complete surgical resection of early-stage NSCLC.

\section{Acknowledgements}

Author contributions: all authors contributed to and approved the final draft of the manuscript. Conception and design: Sumin Shin, Hye Yun Park, Young Mog Shim, Juhee Cho. Experiments and data acquisition: Sumin Shin, Hye Yun Park, Young Mog Shim, Hong Kwan Kim, Yong Soo Choi, Jhingook Kim, Sang-Won Um, Myung Jin Chung, Hojoong Kim, O Jung Kwon, Jae Ill Zo. Analysis and interpretation: Sumin Shin, Hye Yun Park, Hyunkyung Kim, Young Mog Shim, Juhee Cho, Eliseo Guallar, Hong Kwan Kim, Sang-Won Um. Drafting the manuscript: Sumin Shin, Hye Yun Park, Hyunkyung Kim, Young Mog Shim, Hong Kwan Kim, Yong Soo Choi, Jhingook Kim, Sang-Won Um, Hojoong Kim, O Jung Kwon, Jae Ill Zo, Young Mog Shim, Juhee Cho. Critical revision of the manuscript: Sumin Shin, Hye Yun Park, Juhee Cho, Eliseo Guallar, Young Mog Shim.

\section{References}

1 Global Initiative for Chronic Obstructive Lung Disease. Global Strategy for the Diagnosis, Management and Prevention of Chronic Obstructive Pulmonary Disease. www.goldcopd.org Date last accessed: May, 2016. Date last updated: 2016.

2 Nussbaumer-Ochsner Y, Rabe KF. Systemic manifestations of COPD. Chest 2011; 139: 165-173

3 Tockman MS, Anthonisen NR, Wright EC, et al. Airways obstruction and the risk for lung cancer. Ann Intern Med 1987; 106: 512-518.

4 de Torres JP, Bastarrika G, Wisnivesky JP, et al. Assessing the relationship between lung cancer risk and emphysema detected on low-dose CT of the chest. Chest 2007; 132: 1932-1938.

5 Skillrud DM, Offord KP, Miller RD. Higher risk of lung cancer in chronic obstructive pulmonary disease. A prospective, matched, controlled study. Ann Intern Med 1986; 105: 503-507.

6 Young RP, Hopkins RJ, Christmas T, et al. COPD prevalence is increased in lung cancer, independent of age, sex and smoking history. Eur Respir J 2009; 34: 380-386.

7 Zhai R, Yu X, Shafer A, et al. The impact of coexisting COPD on survival of patients with early-stage non-small cell lung cancer undergoing surgical resection. Chest 2014; 145: 346-353.

8 Sekine Y, Behnia M, Fujisawa T. Impact of COPD on pulmonary complications and on long-term survival of patients undergoing surgery for NSCLC. Lung Cancer 2002; 37: 95-101.

9 Sekine $\mathrm{Y}$, Yamada $\mathrm{Y}$, Chiyo $\mathrm{M}$, et al. Association of chronic obstructive pulmonary disease and tumor recurrence in patients with stage IA lung cancer after complete resection. Ann Thorac Surg 2007; 84: 946-950.

10 Han MK, Agusti A, Calverley PM, et al. Chronic obstructive pulmonary disease phenotypes: the future of COPD. Am J Respir Crit Care Med 2010; 182: 598-604.

11 Lee JH, Lee YK, Kim EK, et al. Responses to inhaled long-acting beta-agonist and corticosteroid according to COPD subtype. Respir Med 2010; 104: 542-549.

12 Ueda K, Jinbo M, Li TS, et al. Computed tomography-diagnosed emphysema, not airway obstruction, is associated with the prognostic outcome of early-stage lung cancer. Clin Cancer Res 2006; 12: 6730-6736.

13 Sekine Y, Suzuki H, Yamada Y, et al. Severity of chronic obstructive pulmonary disease and its relationship to lung cancer prognosis after surgical resection. Thorac Cardiovasc Surg 2013; 61: 124-130.

14 Yoshida Y, Kage H, Murakawa T, et al. Worse prognosis for stage IA lung cancer patients with smoking history and more severe chronic obstructive pulmonary disease. Ann Thorac Cardiovasc Surg 2015; 21: 194-200.

15 Berry MF, Jeffrey Yang CF, et al. Impact of pulmonary function measurements on long-term survival after lobectomy for stage I non-small cell lung cancer. Ann Thorac Surg 2015; 100: 271-276.

16 Miller MR, Hankinson J, Brusasco V, et al. Standardisation of spirometry. Eur Respir J 2005; 26: 319-338.

17 Wang Z, Gu S, Leader JK, et al. Optimal threshold in CT quantification of emphysema. Eur Radiol 2013; 23. 975-984.

18 Hersh CP, Make BJ, Lynch DA, et al. Non-emphysematous chronic obstructive pulmonary disease is associated with diabetes mellitus. BMC Pulm Med 2014; 14: 164. 
19 Goldstraw P, Crowley J, Chansky K, et al. The IASLC Lung Cancer Staging Project: proposals for the revision of the TNM stage groupings in the forthcoming (seventh) edition of the TNM Classification of malignant tumours. J Thorac Oncol 2007; 2: 706-714.

20 de Torres JP, Marín JM, Casanova C, et al. Lung cancer in patients with chronic obstructive pulmonary disease incidence and predicting factors. Am J Respir Crit Care Med 2011; 184: 913-919.

21 Smith BM, Pinto L, Ezer N, et al. Emphysema detected on computed tomography and risk of lung cancer: a systematic review and meta-analysis. Lung Cancer 2012; 77: 58-63.

22 DeCamp MM, Blackstone EH, Naunheim KS, et al. Patient and surgical factors influencing air leak after lung volume reduction surgery: lessons learned from the National Emphysema Treatment Trial. Ann Thorac Surg 2006; 82: 197-206.

23 Vestbo J, Prescott E, Almdal T, et al. Body mass, fat-free body mass, and prognosis in patients with chronic obstructive pulmonary disease from a random population sample: findings from the Copenhagen City Heart Study. Am J Respir Crit Care Med 2006; 173: 79-83.

24 Schols AM, Broekhuizen R, Weling-Scheepers CA, et al. Body composition and mortality in chronic obstructive pulmonary disease. Am J Clin Nutr 2005; 82: 53-59.

25 Creutzberg EC, Schols AM, Bothmer-Quaedvlieg FC, et al. Prevalence of an elevated resting energy expenditure in patients with chronic obstructive pulmonary disease in relation to body composition and lung function. Eur J Clin Nutr 1998; 52: 396-401.

26 Lange P, Celli B, Agustí A, et al. Lung-function trajectories leading to chronic obstructive pulmonary disease. N Engl J Med 2015; 373: 111-122.

27 Ministry of Health and Welfare. OECD Health Data 2013. Sejong, Ministry of Health and Welfare, 2013.

28 Asamura H, Suzuki K, Watanabe S, et al. A clinicopathological study of resected subcentimeter lung cancers: a favorable prognosis for ground glass opacity lesions. Ann Thorac Surg 2003; 76: 1016-1022. 